\title{
The Proportional Likelihood Ratio Order for Lindley Distribution
}

\author{
Jarrahiferiz, J. ${ }^{a}$, Mohtashami Borzadaran, G. R. ${ }^{1, a}$, Rezaei Roknabadi, A. H. ${ }^{a}$ \\ ${ }^{a}$ Department of Statistics, Ferdowsi University of Mashhad
}

\begin{abstract}
The proportional likelihood ratio order is an extension of the likelihood ratio order for the non-negative absolutely continuous random variables. In addition, the Lindley distribution has been over looked as a mixture of two exponential distributions due to the popularity of the exponential distribution. In this paper, we first recalled the above concepts and then obtained various properties of the Lindley distribution due to the proportional likelihood ratio order. These results are more general than the likelihood ratio ordering aspects related to this distribution. Finally, we discussed the proportional likelihood ratio ordering in view of the weighted version of the Lindley distribution.

Keywords: Lindley distribution, likelihood ratio order, hazard rate order, mean residual life order, Lorenz order, Laplace order, proportional likelihood ratio order, increasing proportional likelihood ratio order.
\end{abstract}

\section{Introduction}

Stochastic models are usually complex in nature. One of the tools for comparing them is the stochastic ordering of ideas. The start of the idea of stochastic ordering goes back to Lehmann (1955), and after him a significant number of papers and monographs were published. These publications are most commonly found in Shaked and Shanthikumar (2007). The likelihood ratio order also has some nice properties for comparing statistical models. For more details of the history, see the references in page 76 Section 1.C of Shaked and Shanthikumar (2007).

Proportional likelihood ratio order is an extension of the likelihood ratio order that was studied by Ramos-Romero and Sordo-Diaz (2001). In addition, results via the shifted stochastic orders and Laplace order introduced in papers such as Lillo et al. (2001), Ahmed and Kayid (2004), Elbatal (2007), Nanda and Shaked (2008) and extension of the stochastic order for weighted distributions expressed in Bartoszewicz and Skolimowska (2004, 2006) and Neeraj et al. (2008).

Most of statistical properties of the Lindley distribution as a mixture of two exponential distributions are unknown. Ghitany et al. (2008) has shown that the Lindley distribution is a period where model than one based on the exponential distribution and described an application to the waiting time data at a bank. They found that many of the mathematical properties are more flexible than those of the exponential distribution. Sankaran (1970) used a mixing model for the Poisson parameter to generate a mixed Poisson distribution known as the discrete Poisson Lindley distribution. Holgate (1970) and Grandell (1997) obtained results due to the version of mixture distribution such as mixed Poisson. In addition, Ghitany et al. (2008) illustrated a real data application to show the superiority of the Lindley distribution. The ordering aspects previously mentioned are obtained for this distribution.

\footnotetext{
${ }^{1}$ Corresponding author: Department of Statistics, Ordered and Spatial Data Center of Excellence, Ferdowsi University of Mashhad, Mashhad, Iran. E-mail: grmohtashami@um.ac.ir
} 
In this paper, the results related to the proportional likelihood ratio ordering for the Lindley distribution and related criteria are obtained. In addition, extended these, to compare the ordering of various weighted versions of the Lindley distribution are the other ideas of the direction on this work. In the next section, we recall the Lindley distribution, some of the famous stochastic ordering and some results about them. Section 3, contains the results of the proportional likelihood ratio ordering and its shifted version for the Lindley distribution. Proportional likelihood ratio ordering in view of the weighted version of the Lindley distribution is given in Section 4.

\section{Lindley Distribution and Various Ordering}

Mixture distributions with the form $f(x)=\alpha f_{1}(x)+(1-\alpha) f_{2}(x)$ such that $f_{1}$ and $f_{2}$ are two probability distributions and $0 \leq \alpha \leq 1$, have an important role in statistics. Most of phenomena of the nature have a mixture distribution and the statistical analysis of this type of distributions often leads to complicated forms. Lindley $(1958,1965)$ considered types of distribution, and Ghitany et al. (2008) specified distribution with the density,

$$
f(x)=\frac{\theta^{2}}{\theta+1}(1+x) e^{-\theta x}, \quad x>0, \theta>0,
$$

where is a mixture distribution such that $\alpha=\theta /(\theta+1), f_{1}(x)=\theta e^{-\theta x}$ and $f_{2}(x)=\theta^{2} x e^{-\theta x}$ for $x \geq 0$. It is called the Lindley distribution as a mixture of exponential type of gamma distributions. Lindley's model is obtained as a mixture distribution that $\alpha$ depends on the parameter of the distribution, where these type of mixtures are discussed in Glaser (1980) and Gupta and Warren (2001). The corresponding cumulative distribution function(cdf) is,

$$
F(x)=1-\frac{\theta+1+\theta x}{\theta+1} e^{-\theta x},
$$

for $x>0$ and $\theta>0$. Many properties such as moments and related measures, failure rate, mean residual life, mean deviation, Lorenz curve, order statistics, sums, product, and estimation due to Lindley distribution are obtained in Ghitany et al. (2008) that provides a positive reference for some properties of this distribution. Note that this is related to some stochastic ordering due to this distribution. Let $X$ and $Y$ be two independent random variables with cumulative distribution functions $F$ and $G$, probability density functions $f$ and $g$, and hazard rates $r_{F}(\cdot)$ and $r_{G}(\cdot)$, respectively with the support domain $S$. For comparing two random variables via various orderings, we need the following definitions in view of the above arguments. For more details see Shaked and Shanthikumar (2007):

\section{Definition 1.}

(i) $X$ is stochastically smaller than $Y$ (denoted by $X \leq_{s t} Y$ ) if $F(x) \geq G(x)$ for all $x \in S$.

(ii) $X$ is smaller than $Y$ in hazard rate order (denoted by $X \leq_{h r} Y$ ) if $r_{F}(x) \geq r_{G}(x), \forall x \in S$, which is equivalent to $\bar{F}(x) / \bar{G}(x)$ decreases in $x \in S$.

(iii) $X$ is smaller than $Y$ in mean residual life order (denoted by $X \leq_{m r l} Y$ ) if $m_{F}(x) \leq m_{G}(x), \forall x \in S$ where $m_{F}(x)=E(X-x \mid X>x)$.

(iv) The Lorenz order is closely connected to the Lorenz curve, that is defined as follows: suppose $F(x)$ is the distribution function of a non-negative random variable $X$ with finite mean 
$\mu$. Let $F^{-1}$ denotes the inverse of $F$ defined by $F^{-1}(p)=\inf \left\{x: F_{X}(x) \geq p\right\}, p \in[0,1]$, then the Lorenz curve corresponding to $X$ can be defined (Gastwirth, 1971) as,

$$
L_{X}(p)=\frac{1}{\mu} \int_{0}^{p} F^{-1}(t) d t, \quad 0 \leq p \leq 1 .
$$

We say that $X$ is smaller than $Y$ in the Lorenz order (denote by $X \leq_{L} Y$ ) if and only if $L_{X}(p) \geq$ $L_{Y}(p)$ for every $0 \leq p \leq 1$.

Let $X_{(t)}=[t-X \mid X \leq t]$, for fixed $t>0$, when $X$ is the past time of device $X$, can be called inactivity time (past). Ahmad and Kayid (2005) found the characterizations of the reversed hazard rate, the mean inactivity time orderings, decreasing reversed hazard rate, and increasing mean inactivity time classes of the life distributions.

Definition 2. Let $X$ and $Y$ be two non-negative random variables with densities $f$ and $g$, respectively, then,

(i) $X$ is smaller than $Y$ in the reversed hazard rate order (denoted by $X \leq_{R H R} Y$ ) if $X_{(t)} \geq_{s t} Y_{(t)}$ for all $t \geq 0$.

(ii) $X$ is smaller than $Y$ in the mean inactivity time order (denoted by $X \leq_{M I T} Y$ ) if and only if $E\left[X_{(t)}\right] \geq E\left[Y_{(t)}\right]$ for all $t \geq 0$.

(iii) $g(x) / f(x)$ increases over the union of the supports of $X$ and $Y$, (here a/0 is taken to be equal to $\infty$ whenever $a>0$ ). Then $X$ is said to be smaller than $Y$ in the likelihood ratio order (denoted by $X \leq_{l r} Y$ ).

The following notes are due to Lindley distribution in view of ordering:

Theorem 1. (Ghitany et al. 2008) Let $X \sim \operatorname{Lindley}\left(\theta_{1}\right)$ and $Y \sim \operatorname{Lindley}\left(\theta_{2}\right)$. If $\theta_{1}>\theta_{2}$, then $X \leq_{l r} Y$.

Remark 1. It is known that likelihood ratio ordering implies several stochastic ordering such as

$$
X \leq_{l r} Y \Longrightarrow X \leq_{h r} Y \Longrightarrow X \leq_{m r l} Y \text {. }
$$

We have the following theorem in view of the mixtures of distributions:

Theorem 2. Let $X \sim f(x)=\alpha f_{1}(x)+(1-\alpha) f_{2}(x)$ and $Y \sim g(x)=\beta g_{1}(x)+(1-\beta) g_{2}(x)$, where $T_{i} \sim f_{i}$ and $S_{j} \sim g_{j}, i, j=1,2$ are probability density functions, such that $0<\alpha, \beta<1$ and $T_{i} \leq_{l r} S_{j}$, $\forall i, j=1,2$, then $X \leq_{l r} Y$.

Proof: Navarro (2008), considered for pdf $f$ and $g ; \eta_{X}(t)=-f^{\prime}(t) / f(t), \eta_{Y}(t)=-g^{\prime}(t) / g(t)$ and proved that $X \leq_{l r} Y \Longleftrightarrow \eta_{X} \geq \eta_{Y}, \forall t$. Suppose that $h(x)=g(x) / f(x)$, we must prove that $h^{\prime}(x) \geq 0$, assuming we have, $T_{i} \leq_{l r} S_{j}, \forall i, j$, so,

$\eta_{T_{i}} \geq \eta_{S_{j}} \Longrightarrow-f_{i}^{\prime}(x) / f_{i}(x) \geq-g_{j}^{\prime}(x) / g_{j}(x), \forall i, j$. Noting that,

$$
g_{j}^{\prime}(x) f_{i}(x)-f_{i}^{\prime}(x) g_{j}(x) \geq 0,
$$

leads to $h(x)$ is increasing which is equivalent to $X \leq_{l r} Y$. 


\section{Proportional Likelihood Ratio Order and its Shifted Version for the Lindley Distribution}

The likelihood ratio order is stronger than the other stochastic orders like the hazard rate and reversed hazard rate orders, as well as the usual stochastic order, introduced by Ross (1983). This order is an important tool to judge the comparative behavior of the statistical models. Many properties of the likelihood ratio order can be seen in Shaked and Shanthikumar (2007). Ramos-Romero and Sordo-Diaz (2001) introduced an extended version of the likelihood ratio order for non-negative and absolutely continuous random variables (called the proportional likelihood ratio order) to obtain the properties and applications of it as well as its relation with distribution theory.

Proportional likelihood ratio order is discussed in this section for the Lindley distribution and allied aspects. The proportional likelihood ratio order as the extension of this theorem and the shifted proportional likelihood ratio order in view of this distribution is obtained in this section as a new result.

\section{Definition 3.}

(i) If $X$ and $Y$ have interval supports then we say $X$ is smaller than $Y$ in the up likelihood ratio order if $X-x \leq_{l r} Y$ (denote by $X \leq_{l r \uparrow} Y$ ). Thus, we observe that $X \leq_{l r \uparrow} Y$ if and only if,

$$
\frac{g_{Y}(t)}{f_{X}(t+x)} \quad \text { is increasing in } t \in\left(l_{X}-x, u_{X}-x\right) \cup\left(l_{Y}, u_{Y}\right)
$$

for each $x \geq 0$ where $l_{X}$ and $u_{X}$ are left and right endpoints of the support of $X$. Similarly define $l_{Y}$ and $u_{Y}$.

(ii) If $X$ and $Y$ have support $[0, \infty)$. Suppose that, $X \leq_{l r}[Y-x \mid Y>x]$ for all $x \geq 0$, then $X$ is said to be smaller than $Y$ in the down shifted likelihood ratio order (denoted as $X \leq_{l r \downarrow} Y$ ).

Definition 4. Let $X$ and $Y$ be non-negative and absolutely continuous random variable with supports $\operatorname{supp}(X)$ and $\operatorname{supp}(Y)$, respectively. Denote the density function of $X$ and $Y$ by $f$ and $g$, respectively. Suppose that $g(\lambda x) / f(x)$ increases in $x$ for any positive constant $\lambda<1$ over the union of the supports of $X$ and $Y$ (here $a / 0$ is taken to be equal to $\infty$ whenever $a>0$ ). Then we say that $X$ is smaller than $Y$ in the proportional likelihood ratio order (denoted as $X \leq_{p l r} Y$ ).

Ramos-Romero and Sordo-Diaz (2001), obtained results such as if $X \leq_{p l r} Y$ then, $l_{X} \leq l_{Y}$ and $u_{X} \leq u_{Y}$ and $\mu_{X} \leq \mu_{Y}$ or $X \leq_{p l r} Y$ if and only if $X \leq_{l r} a Y$ for all $a>1$. They also showed that if $Y$ has a log-concave density function, then, $X \leq_{l r} Y \Longrightarrow X \leq_{p l r} Y$.

Definition 5. Let $X$ be a continuous non-negative random variable with density $f$. It will be said $X$ is increasing proportional likelihood ratio(IPLR) if $f(\lambda x) / f(x)$ is increasing in $x$ for any positive constant $\lambda<1$.

Next we obtain results for the Lindley distribution in view of the proportional likelihood ratio order as new achievements.

Theorem 3. Let $X \sim \operatorname{Lindley}\left(\theta_{1}\right)$ and $Y \sim \operatorname{Lindley}\left(\theta_{2}\right)$ be two independent random variables. If $\theta_{1}>\theta_{2}$, then, $X \leq_{p l r} Y$.

Proof: Lindley distribution is log-concave, because $\partial^{2} \log f(x) / \partial x^{2}<0$. Via Ramos-Romero and Sordo-Diaz (2001), we know that let $X$ and $Y$ be non-negative and absolutely continuous random 
variables where $Y$ has a log-concave density, then $X \leq_{l r} Y \Longrightarrow X \leq_{p l r} Y$. Thus, let $X \sim \operatorname{Lindley}\left(\theta_{1}\right)$ and $Y \sim \operatorname{Lindley}\left(\theta_{2}\right)$ and $\theta_{1}>\theta_{2}$, then,

$$
X \leq_{l r} Y \Longleftrightarrow X \leq_{p l r} Y .
$$

Remark 2. $X \leq_{l r \uparrow} Y \Longleftrightarrow X \leq_{l r} Y$ via $\log$-concavity of $X$ or $Y$. So under the conditions of the Theorem $3, X \leq_{l r \uparrow} Y$.

Remark 3. We say that $X$ is an increasing failure rate(IFR) random variable, if $\bar{F}$ is log-concave on its support. If $X \sim \operatorname{Lindley}(\theta)$, then, $\partial \log \bar{F}(x) / \partial x=\theta /(\theta+1+\theta x)-\theta$ and $\partial^{2} \log \bar{F}(x) / \partial x<0$, that implies $X$ is IFR. If the random variable $\log X$ is IFR, then $X_{(b, \infty)} \leq_{L} X_{(a, \infty)}$ for all $a<b, a, b \in$ $\operatorname{supp}(X)$. It is hold for the Lindley distribution. If $X \sim \operatorname{Lindley}(\theta)$ then, $X \leq_{l r \uparrow} X$.

Remark 4. We know that let $X_{i}, Y_{i}, i=1,2, \ldots, m$ be independent of random variables that, $X_{i} \sim$ Lindley $\left(\theta_{1}\right)$ and $Y_{i} \sim \operatorname{Lindley}\left(\theta_{2}\right), i=1,2, \ldots, m$, where $\theta_{1}>\theta_{2}$, then, $\sum_{i=1}^{m} X_{i} \leq_{l r} \sum_{i=1}^{m} Y_{i}$. Also, let $\psi$ be an increasing (decreasing) function, then, $\psi\left(X_{i}\right) \leq_{l r}\left(\geq_{l r}\right) \psi\left(Y_{i}\right), i=1,2, \ldots, m$.

Remark 5. We can have a theorem similar to Theorem 1 in view of the proportional likelihood ratio order, then its calculations are a little complicated.

In here, we study some properties due to shifted orders for Lindley distributions.

Definition 6. Let $X$ and $Y$ be continuous random variables with densities $f$ and $g$, respectively, such that $X-x \leq_{p l r} Y, \quad \forall x \geq 0$. Then, we say that $X$ is smaller than $Y$ in the up shifted proportional likelihood ratio and denotes as $X \leq_{p l r \uparrow} Y$. In fact, $X \leq_{\text {plr } \uparrow} Y$ if and only if $g(\lambda(t+x)) / f(t)$ is increasing on $t \in\left(l_{x} / \lambda-x, u_{x} / \lambda-x\right) \cup\left(l_{y}, u_{y}\right)$ for $x \geq 0$ and $0<\lambda<1$.

Theorem 4. Let $X$ and $Y$ be two non-negative and continuous random variables, respectively, then

a) $X \leq_{p l r \uparrow} Y \Longleftrightarrow X \leq_{l r \uparrow} a Y, \quad \forall a>1$.

b) If $Y$ have a log-concave density, then, $X \leq_{l r \uparrow} Y \Longrightarrow X \leq_{p l r \uparrow} Y$.

Remark 6 . Let $X \sim \operatorname{Lindley}\left(\theta_{1}\right)$ and $Y \sim \operatorname{Lindley}\left(\theta_{2}\right)$ be two independent random variables. If $\theta_{1}>\theta_{2}$, then $X \leq_{p l r \uparrow} Y$.

Definition 7. Let $X$ be continuous random variable with density $f$, we say that $X$ have up increasing proportional likelihood ratio(UIPLR) if $f_{X}(\lambda(t+x)) / f_{X}(t)$ increasing on $t$ where $0<\lambda<1$.

Remark 7. If $X \sim \operatorname{Lindley}(\theta)$, then $X$ is UIPLR and

$$
X \text { is IPLR } \Longleftrightarrow X \leq_{l r} a X, \quad \forall a>1 \Longleftrightarrow X \leq_{p l r} X .
$$

Remark 8. For a random variable $X$ with distribution function $F$, the Laplace-Stieltjes transform of $F_{X}$ is given by $L_{* X}(s)=\int_{0}^{\infty} e^{-s u} d F_{X}(u), s>0$.

Given two random variables $X$ and $Y$, then $X$ is said to be smaller than $Y$ in the Laplace transform $\operatorname{order}\left(X \leq_{L t} Y\right)$ if $L_{* X}(s) \geq L_{* Y}(s)$ for all $s>0$. In addition, let $L_{X}^{*}(s)=\int_{0}^{\infty} e^{-s u} \bar{F}_{X}(u) d u=$ $\left\{1-L_{* X}(s)\right\} / s$, then $X \leq_{L t} Y \Longleftrightarrow L_{X}^{*}(s) \leq L_{Y}^{*}(s)$ for all $s>0$ (Shaked and Shanthikumar, 2007). 
Definition 8. Let $X$ and $Y$ be two non-negative random variables. $X$ is said to be smaller than $Y$ in the Laplace transform order of residual life $\left(X \leq_{L t-r l} Y\right)$ if $X_{t} \leq_{L t} Y_{t}$ for all $t \in\left(0, l_{X}\right) \cap\left(0, l_{Y}\right)$ where $X_{t}=[X-t \mid x>t], l_{X}=\sup \{t, F(t)<1\}$ and $L_{X_{t}}^{*}(s)=\int_{t}^{\infty}\left\{e^{-s u} \bar{F}_{X}(u) d u\right\} /\left\{e^{-s t} \bar{F}(t)\right\}, s>0$.

Theorem 5. (Belzunce et al., 1999) Let $X$ and $Y$ be two continuous random variables, then $X \leq_{L t-r l}$ $Y \Longleftrightarrow \int_{t}^{\infty} e^{-s u} \bar{F}_{X}(u) d u / \int_{t}^{\infty} e^{-s u} \bar{F}_{Y}(u) d u$ is decreasing in $t \in\left(0, l_{X}\right) \cap\left(0, l_{Y}\right)$ for all $s>0$.

Remark 9. The following implication of ordering show links between them:

$$
\begin{gathered}
X \leq_{l r} Y \Longrightarrow X \leq_{h r} Y \Longrightarrow X \leq_{L t-r l} Y \Longrightarrow X \leq_{m r l} Y \\
\Downarrow \\
X \leq_{L t} Y .
\end{gathered}
$$

Remark 10. On noting the theorem that is mentioned in Ahmad and Kayid (2005), let $X$ and $Y$ be two continuous nonnegative random variables.

(i) For all $t \geq 0, \quad X \leq_{R H R} Y \Longleftrightarrow X_{(t)} \geq_{L t} Y_{(t)}$.

(ii) If $X$ and $Y$ have differentiable $M I T$ functions $\mu$ and $\beta$, respectively. Suppose that $\mu(t) / \beta(t)$ is increasing in $t$. Then, $X \leq_{M I T} Y \Longleftrightarrow X \leq_{R H R} Y$. So,

$$
\begin{gathered}
X \leq_{p l r} Y \Longrightarrow X \leq_{l r} Y \Longrightarrow X \leq_{R H R} Y \Longrightarrow X \leq_{M I T} Y \\
X_{(t)} \geq_{L t} Y_{(t)} .
\end{gathered}
$$

Applying to Lindley distributions leads to the following result :

Theorem 6. Let $X \sim \operatorname{Lindley}\left(\theta_{1}\right)$ and $Y \sim \operatorname{Lindley}\left(\theta_{2}\right)$ be two random variables, then

$$
\theta_{1}>\theta_{2} \Longleftrightarrow X \leq_{L t} Y \Longleftrightarrow L_{X}(s) \geq L_{Y}(s) \text {. }
$$

Proof: It is concluded from Remark 9 , that $X \leq_{l r} Y \Longrightarrow X \leq_{L t} Y$.

\section{Proportional Likelihood Ratio Order for Weighted Version of Lindley Distribution}

Consider a mechanism generating a non-negative random variable $X$ with density function $f($.$) . Let$ $w\left(\right.$.) be a non-negative function with finite non zero expectation. Define a random variable $\hat{X}_{w}$ with density function as $\hat{X}_{w} \sim w(x) f(x) / E(w(X))$, where $\hat{X}_{w}$ is called weighted random variable corresponding to $f(x)$ (see Rao 1965). Special cases of it, choosing various values for $w$ lead to various famous distributions in statistics.

We will concentrate on proportional likelihood ratio order via weighted distribution and specially weights such as size biased, order statistics, record value, residual life time of a stationary renewal process, selection samples, hazard rate, reversed hazard rate, proportional hazard model, reversed proportional hazard model and probability weighted moments are some special cases of weighted families.

Theorem 7. (Bartoszewicz and Skolimowska, 2006) Let $F$ be an absolutely continuous distribution. If $w$ is increasing (decreasing), then $X \leq_{l r}\left(\geq_{l r}\right) \hat{X}_{w}$. 
Remark 11. On noting that Lindley distribution is IPLR, it is clear that $X \leq_{p l r} \hat{X}_{w}$, where $X \sim$ Lindley $(\theta)$ when $w$ is increasing.

Based on arguments of Theorem 7, the following theorems are clear.

Theorem 8. If $X \sim$ Lindley $(\theta)$ and $w(x)$ be an increasing (decreasing) function of $x$, then $X \leq_{p l r}$ $\left(\geq_{p l r}\right) \hat{X}_{w}$ where $\hat{X}_{w} \sim g(x)=w(x) f(x) / E(w(X))$.

Theorem 9. Let $X \sim \operatorname{Lindley}\left(\theta_{1}\right)$ and $Y \sim$ Lindley $\left(\theta_{2}\right)$ be two independent random variables, such that $\theta_{1}>\theta_{2}$ then if $\hat{X}_{w} \sim w(x) f(x) / E(w(X))$ and $\hat{Y}_{w} \sim w(x) g(x) / E(w(X))$ and $w(\lambda x) / w(x)$ is increasing, then $\hat{X}_{w} \leq_{p l r} \hat{Y}_{w}$.

Theorem 10. Let $X$ and $Y$ be two continuous random variables with interval support and weighted distribution of Lindley $(\theta)$ with weights $w_{1}$ and $w_{2}$ respectively. If $\lambda w_{1}^{\prime}(\lambda x) / w_{1}(\lambda x)>w_{2}^{\prime}(x) / w_{2}(x)$, then $X \leq_{p l r} Y$.

Proof: Let $X \sim g_{1}(x)=w_{1}(x) /\left\{E w_{1}(X)\right\} * f(x)$ and $Y \sim g_{2}(x)=w_{2}(x) /\left\{E w_{2}(X)\right\} * f(x)$ where $f(x)=$ $\theta^{2} /(\theta+1) *(1+x) e^{-\theta x} . f(\lambda x) / f(x)=(1+\lambda x) /(1+x) * e^{-\theta x(\lambda-1)}$ is increasing in $x$ for any positive constant $\lambda<1$. So, $X \sim \operatorname{Lindley}(\theta)$ is IPLR. It is clear that $g_{1}(\lambda x) / g_{2}(x)=E w_{2}(X) / E w_{1}(\lambda X) *$ $w_{1}(\lambda x) / w_{2}(x) * f(\lambda x) / f(x)$ increasing with respect to $x$ for $\lambda<1$.

Remark 12. For $w_{1}(x)=x^{i}[F(x)]^{j}[\bar{F}(x)]^{k}$ and $w_{2}(x)=x^{i^{\prime}}[F(x)]^{j^{\prime}}[\bar{F}(x)]^{k^{\prime}}$, we have $i / x+j \lambda f(\lambda x) /$ $F(\lambda x)-k \lambda f(\lambda x) / \bar{F}(\lambda x)>i^{\prime} / x+j^{\prime} f(x) / F(x)-k^{\prime} f(x) / \bar{F}(x)$ leads to $X \leq_{p l r} Y$. Now we consider some special cases:

(1) $i=i^{\prime}=0$ implies $j \lambda f(\lambda x) / F(\lambda x)-k \lambda f(\lambda x) / \bar{F}(\lambda x)>j^{\prime} f(x) / F(x)-k^{\prime} f(x) / \bar{F}(x)$ leads to $X \leq_{p l r} Y$.

(2) If $i=i^{\prime}=0, k=n-j-1, k^{\prime}=m-j^{\prime}-1$ then $j \lambda f(\lambda x) / F(\lambda x)-(n-j-1) \lambda f(\lambda x) / \bar{F}(\lambda x)>$ $j^{\prime} f(x) / F(x)-\left(m-j^{\prime}-1\right) f(x) / \bar{F}(x)$ leads to $X \leq_{p l r} Y$.

(3) If $i=i^{\prime}=j=j^{\prime}=0$ so, $k \lambda f(\lambda x) / \bar{F}(\lambda x)<k^{\prime} f(x) / \bar{F}(x)$ results that $X \leq_{p l r} Y$.

(4) $i=i^{\prime}=k=k^{\prime}=0$ implies that $j \lambda f(\lambda x) / F(\lambda x)>j^{\prime} f(x) / F(x)$ is a condition that leads to $X \leq_{p l r} Y$.

The conclusions that for two weights such as probability weighted moments, order statistics, proportional hazard and reversed proportional hazard are mentioned in this remark.

Remark 13. For $w_{1}(x)=[-\ln \bar{F}(x)]^{i}[-\ln F(x)]^{j}$ and $w_{2}(x)=[-\ln \bar{F}(x)]^{i^{\prime}}[-\ln F(x)]^{j^{\prime}}$ we have $f(\lambda x) /$ $\bar{F}(\lambda x)[i \lambda /\{-\ln \bar{F}(\lambda x)\}]-f(\lambda x) / F(\lambda x)[j \lambda /\{-\ln F(\lambda x)\}]>f(x) / \bar{F}(x)\left[i^{\prime} /\{-\ln \bar{F}(x)\}\right]-f(x) / F(x)\left[j^{\prime} /\right.$ $\{-\ln F(x)\}]$ leads to $X \leq_{p l r} Y$. Note that,

(1) if $i=i^{\prime}=0$, then "upper record with weight $w_{1}(x)$ " $\leq_{p l r}$ "upper record with weight $w_{2}(x)$ ",

(2) when $j=j^{\prime}=0$, then, "Lower record with weight $w_{1}(x)$ " $\leq_{p l r}$ "Lower record with weight $w_{2}(x)$ ".

Remark 14. Note that $\left(i=i^{\prime}=0, k=n-j-1, k^{\prime}=m-j^{\prime}-1\right)$ is equivalent to $X_{j: n} \leq_{p l r} Y_{j^{\prime}: m}$. Via Shaked and Shanthikumar (2007), we know that if $X_{i} \sim \operatorname{Lindley}\left(\theta_{i}\right) ; i=1,2, \ldots, m$ such that $\theta_{1}>\theta_{2}>\cdots>\theta_{m}$, then $X_{(k-1: m)} \leq_{l r} X_{(k: m)}, 2 \leq k \leq m$ and $X_{(k-1: m-1)} \leq_{l r} X_{(k: m)}, 2 \leq k \leq m$ can be discussed in view of the proportional likelihood ratio order. 
Theorem 11. Let $X$ be a continuous random variable with interval support and weighted distribution of $Y \sim \operatorname{Lindley}(\theta)$ with weight $w$. Then, $X \leq_{\text {plr }} Y$.

Proof: Via the arguments of the previous theorem on noting that $w(x)=w_{1}(x)$ and $w_{2}(x)=1$, it is obvious that $w_{2}^{\prime}(x)=0$, and $\lambda w^{\prime}(\lambda x) / w(\lambda x)>0$ implies increasing property of $w$.

Remark 15. In previous theorem when $w(x)=x^{i}[F(x)]^{j}[\bar{F}(x)]^{k} e^{l x}$, then, $i F(x) \bar{F}(x)+j x f(x) \bar{F}(x)+$ $l>k x f(x) F(x), \forall x \Longrightarrow X \leq_{p l r} Y$.

- When $w(x)=e^{l x}, l>0 \Longrightarrow X \leq_{p l r} Y$.

- For order statistics case, $w(x)=[F(x)]^{j}[\bar{F}(x)]^{n-j-1}$ so, $F(x)<j / n, \forall x \Longrightarrow X \leq_{p l r} Y$.

- $w(x)=[F(x)]^{j}$ without any condition implies $X \leq_{p l r} Y$, but $w(x)=[\bar{F}(x)]^{k}$ does not imply always $X \leq_{p l r} Y$.

\section{Conclusion}

We have recalled the Lindley distribution as a mixture of two exponential distributions and some of the famous stochastic ordering such as likelihood ratio order, hazard rate order, Laplace order and Lorenz order. Then studied the properties of the proportional likelihood ratio ordering and its shifted version for this distribution that are held for all weaker ordering (assuming $\lambda=1$ and $x=0$ ). Finally we considered the weighted version of the Lindley distribution and obtained the results, particularly for some weights such as upper record, lower record, weighted moment probability, proportional hazard rate, and proportional reversed hazard rate.

\section{Acknowledgment}

Partial support from the Ordered and Spatial Data Center of Excellence of Ferdowsi University of Mashhad is acknowledged. In addition, the authors are grateful to the Associate Editor and the referees for suggestions and comments that have improved this paper.

\section{References}

Ahmed, H. and Kayid, M. (2004). Preservation properties for the Laplace transform ordering of residual lives, Statistical Papers, 45, 583-590.

Ahmad, I. A. and Kayid, M. (2005). Characterizations of the RHR and MIT orderings and the DRHR and IMIT classes of life distribution, Probability in the Engineering and Informational Sciences, $19,447-461$.

Bartoszewicz, J. and Skolimowska, M. (2004). Stochastic ordering of weighted distributions, University of Wroclaw. Report No. 143, available at www.math.uni.worc.pl/mathbank/ preprint.

Bartoszewicz, J. and Skolimowska, M. (2006). Preservation of classes of life distributions and stochastic orders under weighting, Statistics and Probability Letters, 76, 587-596.

Belzunce, F., Ortega, E. and Ruiz, J. M. (1999). The Laplace order and ordering of residual lives, Statistics and Probability Letters, 42, 145-156.

Elbatal, I. (2007). The Laplace order and ordering of reversed residual life, Applied Mathematical Sciences, 36, 1773-1788.

Gastwirth, J. L. (1971). A general definition of the Lorenz curve, Econometrica, 39, 1037-1039. 
Ghitany, M. E., Atieh, B. and Nadarajah, S. (2008). Lindley distribution and its application, Mathematics and Computers in Simulation, 78, 493-506.

Glaser, R. E. (1980). Bathtub and related failure rate characterizations, Journal of the American Statistical Association, 75, 667-672.

Grandell, J. (1997). Mixed Poisson Processes, Chapman and Hall, London.

Gupta, R. C. and Warren, R. (2001). Determination of change points of non-monotonic failure rates, Communications in Statistics-Theory and Methods, 30, 1903-1920.

Holgate, P. (1970). The modality of some compound Poisson distribution, Biometrika, 57, 666-667.

Lehmann, E. L. (1955). Ordered families of distributions, Annals of Mathematical Statistics, 26, 399-419.

Lillo, R. E., Nanda, A. K. and Shaked, M. (2001). Some shifted stochastic order, Recent Advances in Reliability Theory, Methodology, Practice and Inference, 85-103.

Lindley, D. V. (1958). Fiducial distribution and Bayes theorem, Journal of the Royal Statistical Society, 20, 102-107.

Lindley, D. V. (1965). Introduction to Probability and Statistics from a Bayesian Viewpoint, Cambridge University Press, New York.

Nanda, A. K. and Shaked, M. (2008). Partial ordering and ageing properties of order statistics when the sample size is random: A brief review, Communications in Statistics- Theory and Methods, 37, 1710-1720.

Navarro, J. (2008). Likelihood ratio ordering of order statistics, mixtures and systems, Statistical of Planning and Inference, 138, 1242-1257.

Neeraj, M., Nitin, G. and Ishwari, D. D. (2008). Preservation of some aging properties and stochastic order by weighted distributions, Communications in Statistics-Theory and Methods, 37, 627644.

Ramos-Romero, H. M. and Sordo-Diaz, M. A. (2001). The Proportional likelihood ratio order and applications, Questiio, 25, 211-223.

Rao, C. R. (1965). On discrete distributions arising out of methods of ascertainment, Classical and Contagious Discrete Distributions, G. Patil Ed., Pergamon Press and Statistical Publishing Society, Calcutta, 320-332.

Ross, S. M. (1983). Stochastic Processes, Wiley, New York.

Sankaran, M. (1970). The discrete Poisson-Lindley distribution, Biometrics, 26, 145-149.

Shaked, M. and Shanthikumar, J. G. (2007). Stochastic Orders, Springer, New York. 